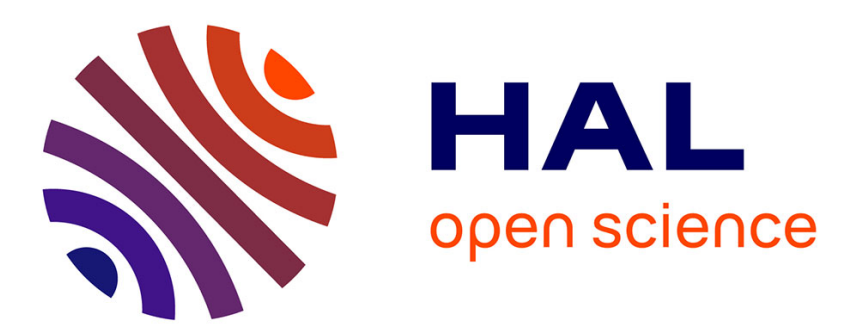

\title{
Consequences of drafting on human locomotion: benefits on sports performance
}

\author{
Christophe Hausswirth, Jeanick Brisswalter
}

\section{To cite this version:}

Christophe Hausswirth, Jeanick Brisswalter. Consequences of drafting on human locomotion: benefits on sports performance. International Journal of Sports Physiology and Performance, 2008, 3 (1), pp.315. 10.1123/ijspp.3.1.3 . hal-01697006

\section{HAL Id: hal-01697006 https://hal-insep.archives-ouvertes.fr/hal-01697006}

Submitted on 30 Jan 2018

HAL is a multi-disciplinary open access archive for the deposit and dissemination of scientific research documents, whether they are published or not. The documents may come from teaching and research institutions in France or abroad, or from public or private research centers.
L'archive ouverte pluridisciplinaire $\mathbf{H A L}$, est destinée au dépôt et à la diffusion de documents scientifiques de niveau recherche, publiés ou non, émanant des établissements d'enseignement et de recherche français ou étrangers, des laboratoires publics ou privés. 


\title{
Consequences of drafting on human locomotion: benefits on sports performance
}

\author{
Hausswirth Christophe ${ }^{1}(\bowtie)$ \& Brisswalter Jeanick ${ }^{2}$ \\ (article paru dans: International Journal of Sports Physiology and Performance, vol. 3, $n^{\circ} 1$, march 2008, pp. 3-15)
}

${ }^{1}$ Institut National du Sport et de l'Education Physique (INSEP),

Laboratory of Biomechanics and Physiology

11 Avenue du Tremblay, Paris 75012, France.

${ }^{2}$ Sport Ergonomy and Performance Laboratory, EA 3162, University of Toulon-Var, Av. De l'Université, BP 20132, 83957 La Garde Cedex - France

Corresponding author: $(\bowtie)$

HAUSSWIRTH Christophe, Ph.D.,

${ }^{1}$ Institut National du Sport et de l'Education Physique (INSEP),

Laboratory of Biomechanics and Physiology

11 Avenue du Tremblay, Paris 75012, France.

$\triangle$ Email: christophe.hausswirth@insep.fr

Tel: +33141744385

Fax: +33141744535 


\section{INTRODUCTION}

In many endurance sports, a large part of an athlete's external power is used to overcome drag. This force has two components: friction and pressure drag. When the athlete's velocity is very low, friction drag dominates. In normal sports activities, pressure drag dominates. However, frictional drag is influential in the velocity range in which the airstream changes from laminar to turbulent, which on the other hand depends on the roughness of the athlete's clothing.

The term 'drafting' is mainly used in sports physiology and biomechanics to describe the tactic of performing a mode of activity in sheltered position. Therefore, the growing success and impact of merging endurance sports where athletes could take advantage in drafting raised renewed questions on the physiological characteristics and mechanisms regulating the human locomotion in sports like running (Pugh, 1971), cycling (Kyle, 1979), speed-skating (Rundell, 1996), swimming (Chatard \& Wilson, 2003), triathlon (Hausswirth et al. 1999). The athlete who drafts continuously during the race may therefore reach a better position than would normally be in line with his individual physiological capacities. For this reason, many athletes attempt to position themselves behind athletes of the same or slightly better ability.

\section{Benefits of drafting in water activities}

\section{$\checkmark$ Consequences on physiological aspects of performance}

Drafting while swimming front crawl, i.e. swimming directly behind or at the side of another swimmer, is mainly used in triathlon races or open-water swims. Drafting allows the swimmer to reduce the energy cost of swimming propulsion and hence gain time for swimming at maximal speed (Chatard et al. 1998). During swimming, hydrodynamic drag could be reduced when swimming in a drafting position. The effects of drafting during short swimming bouts have been widely studied in the recent literature (Basset et al., 1991; Chollet et al. 2000, Millet et al. 2000). The main factor of decreased body drag with drafting seems to be the depression made in the water by the lead swimmer (Chatard \& Wilson, 2003). This low pressure behind the lead swimmer decreases the pressure gradient from the front to the back of the following swimmer, hence facilitating his displacement.

In submaximal conditions, precisely at an intensity of $95 \%$ of maximal speed over a 549-m swim, Basset et al. (1991) showed that drafting affected the metabolic responses to swimming. Oxygen uptake was reduced by $8 \pm 12 \%$, blood lactate concentration by $33 \pm 17 \%$, heart rate by 
$7.3 \%$ after 400 -m (cf. Figure 1), and the rate of perceived exertion by $21 \pm 10 \%$. The lower resistive body drag (passive drag) forces encountered by the swimmers at maximum speed are responsible for the observed metabolic change (Chatard et al. 1998). As elite front crawl swimmers, Millet et al. (2000) suggested that triathletes can use two kick rhythms (two-or sixbeat) during the swim part of a triathlon. Thus, when drafting, they may follow a lead subject swimming with either a two- or a six-beat kick. A six-beat kick creates lots of bubbles and turbulences, making drafting less easy than when sheltering a two-beat kick. However, these authors showed the kick rhythm of the lead did not influence the metabolic responses of the draftee swimmer.

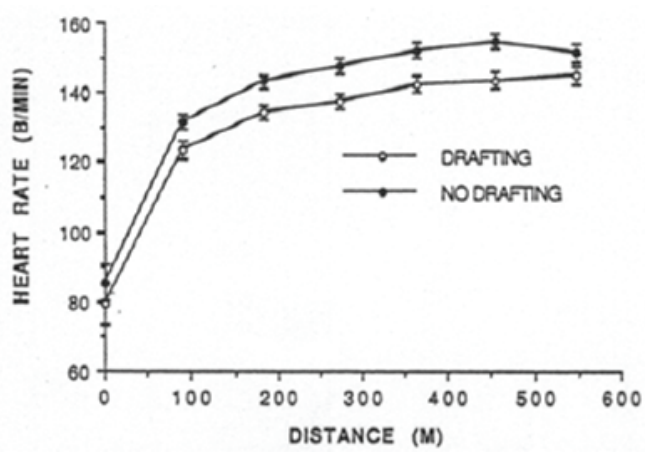

Figure 1 - Heart rate responses (mean \pm SE) to a 549-m swim (95\% maximal swim velocity), with and without drafting. There are significant differences between conditions at 100-600 m $(P<0.05)$, (adapted from Basset et al. 1991)

Studies focusing on the effects of swimming on subsequent cycling performance are restricted (Delextrat et al. 2003; Kreider et al 1988; Laursen et al. 2000). However, despite the lack of experimental studies, recent reviews on triathlon determinants highlighted that the metabolic demand induced by swimming could have detrimental effects on subsequent cycling adaptations (e.g. Bentley et al. 2002). Experimental studies on the effect of prior swimming on subsequent cycling performance have led contradictory results. Kreider et al. (1988) have found that an 800-m swimming bout resulted in a significant decrease in power output $(17 \%)$ during a subsequent 75-min cycling exercise. More recently, Delextrat et al. (2003) have observed a significant decrease in cycling efficiency (17.5\%) after a $750-\mathrm{m}$ swim conducted at a sprint triathlon competition pace when compared with an isolated cycling bout. Actually, in an elite Olympic distance triathlon the strategy of drafting during the cycling part can influence the 
energy demands of this section as well as swimming and running strategies (Bentley et al. 2002). Delextrat et al. (2003) demonstrated the decrease in metabolic load associated with swimming in a drafting position involved two main modifications in physiological parameters during subsequent cycling. Firstly, oxygen uptake kinetics, at the onset of cycling, were significantly slowed when the prior swimming bout was performed in a drafting position (slower time constant) compared with swimming alone. Secondly, a significantly higher cycling efficiency $(+4.8 \%)$, measured at steady-sate level, was observed in the drafting condition compared with the isolated swim. This improvement in cycling efficiency could be mainly accounted for by the lower swimming relative intensity involving a lower state of fatigue in the muscles of the lower limbs at the beginning of the subsequent cycling session. Consequently, the authors suggested that the increase in cycling efficiency could lead to an improvement in the overall performance during a triathlon. It should be noted that the possibility for athletes and coaches to put the results of this study into practice could be limited by the lack of cycling training of subjects tested and by the difference between intensity and duration of the cycling trials and the metabolic load encountered during a sprint distance triathlon (Hausswirth et al. 1999). Because cycling experience could lead to a lower variability in energy cost of locomotion, more training in cycling would be associated with a lower benefit of drafting. In addition to this study, a more recent experiment conducted by Bentley et al. (2007) compared the affects of drafting or a reduction of exercise intensity during swimming on the power output sustained during a subsequent cycle time trial. They found that the power output during a 20 -min time trial in cycling was significantly lower after a $400-\mathrm{m}$ all-out freestyle swimming at either $90 \%$ of this velocity or in a drafting situation. The authors showed no significant difference in the power output during cycling performance after swimming at $90 \%$ or in a drafting position. Thus, they demonstrated that whilst swimming may affect cycling performance, drafting results in a similar performance response during cycling. This could have straight recommendations for training approach in triathlon and/or the strategy to adopt during world cup triathlon event.

Over aquatic sports are commonly studied in order to optimize physiologic performance and to apply scientific basis into the daily practice. For example, a common technique employed in flatwater kayak and canoe races is "wash riding", in which a paddler positions his boat on the wake of a leading boat and, at a strategic moment, drops off the wake to sprint ahead. It was hypothesized by Gray et al. (1995) that this manoeuver was energy efficient, analogous to 
drafting in cycling. This study shows that, in highly trained male kayak paddlers examined during steady-state exercise at 10,000 meter race pace $\left(3.7 \mathrm{~m} \cdot \mathrm{s}^{-1}\right)$, a significant decrease $(-11 \%)$ in energy consumption during wash riding was found compared to non-wash riding, then delaying the onset of fatigue. This finding has implications for the design of training programs and competitive strategy plans for water kayak racing, suggested that practice of wash riding can reduce energy expenditure under speeds similar to those encountered in competitive events.

\section{- Consequences on biomechanical aspects of performance}

The distance adopted by the drafting in swimming appears to be a consistent parameter which could be linked to overall swimming performance. Chatard et al. (2003) investigated the effect of the distance (from 0 to $150 \mathrm{~cm}$ ) separating the lead and the draft swimmer on the metabolic responses of the drafting swimmer performing a 4-min swim in a flume at $95 \%$ of their best $1500-\mathrm{m}$ velocity. They showed that the optimum drafting position was in the 0 - to 50 $\mathrm{cm}$ range behind another swimmer, although a significant reduced metabolic response persisted at the 100- and $150-\mathrm{cm}$ distances. Results demonstrated a reduction in oxygen uptake by $11 \%$ and in stroke rate by $6 \%$, whereas stroke length was increased by $6 \%$ at the optimal drafting distance of $50-\mathrm{cm}$. This result confirmed the average $60-\mathrm{cm}$ distance spontaneously adopted by drafters in high level triathlon (Millet et al. 2000). An over important result of this study was the drafting distance of $150-\mathrm{cm}$ which elicited about $10 \%$ benefits in metabolic cost whereas drafters had a $20 \%$ reduction in drag. This observation could have practical application especially in pool-based training and/or open-water competition like in long-distance swimming or triathlon. However, the context of competition put swimmers sometimes in side-drafting situation and not only in behind-drafting position. The optimum position when $100-\mathrm{cm}$ to the side was $100-\mathrm{cm}$ behind the lead swimmer with the draftee's head located at a level of the hip of the leader (Chatard et al. 2003). This study was the first to demonstrate that swimming beside another swimmer is beneficial in terms of reduction of drag. However, the reduction in resistive drag was only one third of the reduction in drag when drafting immediately behind the lead swimmer. Then, many studies have showed that swimming behind another swimmer in a race is advantageous (Basset et al. 1991; Chatard et al. 2003; Chollet et al. 2000; Millet et al. 2000). The gain in performance was higher for the faster swimmers (Chatard et al. 1998): this point appears to be inconsistent with the gain reduction in drag observed with velocity. This 
explanation put forward is that the faster triathletes are also the better swimmers and thus could take more benefits from the drafting situation because of their better swimming skills. Thus, drafting technique should be increased in training in order to learn more skills and optimize the physiological benefits afforded by a drafting situation. Another parameter which is taken into consideration by triathletes is the wearing of wet suit. Delextrat et al. (2003) demonstrated that the significant effect of drafting previously reported in the scientific literature was observed even though subjects wear a wet suit but the stroke characteristics (stroke rate and stroke length) were not significantly different in drafting position during a 750-m where the draftees wear a wet suit. Consequentltly, the authors demonstrated the influence in swimming alone or in a sheltered position behind a leader on pedal rate during the subsequent cycling for the triathletes; a significant pedal rate of 5.6\% was observed in cycling when swimming was done before with drafting. The authors reported that this previous situation could have involved an increased blood flow to the muscles of the lower limbs. In that context of swimming, it has been also reported that the use of wet suit induced significant decreases in active drag (from 12\% to 16\%) among different swimming speeds (Chatard \& Millet, 1996). These authors showed specifically that swimming behind a leader resulted in an increase in swimming velocity (by 3.2\%, i.e. 20-m benefits over a 400-m) and stroke length, and a reduction in stroke frequency. They found that the gain of performance was related to the ability of the swimmer and his skinfold thickness, with faster and leaner swimmers achieving a greater gain. The higher gain in performance observed for leaner subjects is consistent with the drag varaiations measured by Chatard et al. (1998). Indeed, swimmers with a greater skinfold thickness tend naturally to adopt a more horizontal position because of a better floating posture, and thus have a lower drag than leaner subjects (Pendergast et al., 1977). More recently, Chollet et al. (2000) demonstrated that the performance increased from $1.34 \mathrm{~m} . \mathrm{s}^{-1}$ to $1.39 \mathrm{~m} . \mathrm{s}^{-1}$ when swimmers drafted the leader during a 400-m (Figure 2). Moreover, the authors concluded that drafting also contributes to stabilize the stroke parameters such as stroke frequency and stroke length during swimming (see Figure 2). On the contrary observed in the no-draft situation in swimming, the stroke frequency and the stroke length decreased throughout the 400-m, phenomenon probably due to the acute fatigue developed when swimmers are not sheltered behind another. Chollet et al. (1997) studying 442 swimmers demonstrated that the best swimmers were characterized by their capacity to maintain higher consistency of technique and velocity throughout the race than their counterparts. In the 
same way, improvements in stroke parameters such as stroke length have been also observed in drafting kayaking (Gray et al. 1995); it was interesting to note in this study that, while the kayak velocity increased significantly during the wash riding (i.e. drafting situation) trial, the stroke rate of the paddlers was significantly reduced. This indicates that there was likely a change in the stroke mechanics employed by the paddlers.
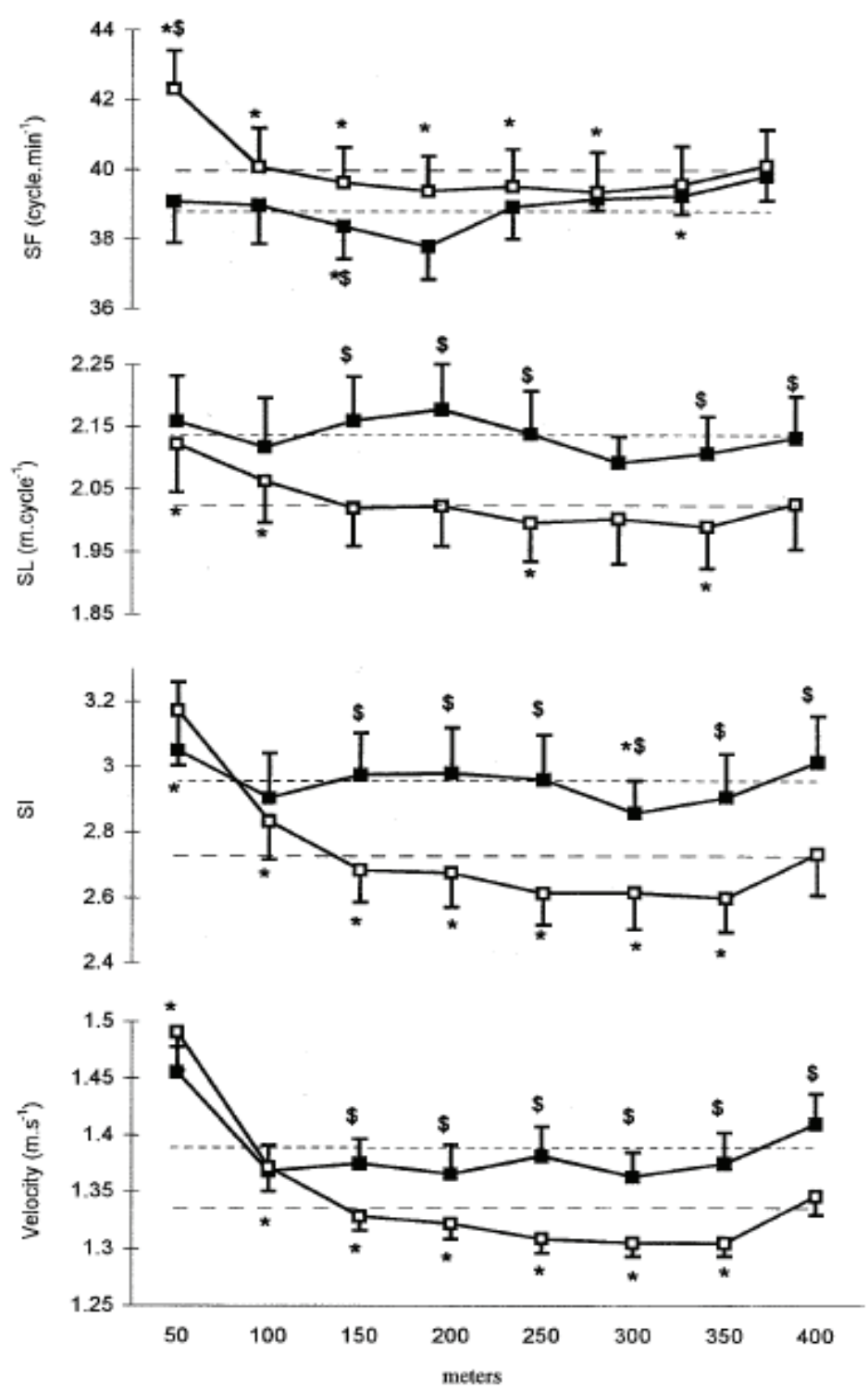

Figure 2 - Differences and variations in swimming velocity, stroke index $(S I)$, stroke length $(S L)$ and stroke frequency $(S F)$ during the drafting (filled squares) and non-drafting (open squares) $400-\mathrm{m}$ swim. ${ }^{\$} P<0.05$ : differences in drafting versus non-drafting conditions; ${ }^{*} P<0.05$ : differences between each $50-\mathrm{m}$ value and mean 400-m in drafting (---) and non-drafting conditions (-) (from Chollet et al. 2000). 
All experimental studies focusing on aquatic sports and its physiological and biomechanical specificities emphasized the high benefits in the drafting process in which 'athlete' could take advantage in increasing his performance. However, drafting technique and drafting ability need to be integrate into daily practical training programs in order to adopt the most efficient position during competitions, and then therefore reach a better position than would normally be in line with his individual physiological and biomechanical capacities.

\section{Benefits of drafting in land activities}

\section{\Consequences on physiological aspects of performance}

During individual road cycling events, it is possible to very accurately predict performance given knowledge of the mass of the system (bicycle and rider), its aerodynamic characteristics, and the athlete's physiological qualities (Di Prampero, 2000; Olds, 2001). During multiple cyclist events riders have the opportunity to draft one another. Drafting is known to afford less physiologically capable individuals the ability to maintain the pace of their more capable counterparts and, in doing so, adds complexity to the prediction of racing performance. In this context the magnitude of the drafting effect in cycling can be impressive. McCole et al. (1990) demonstrated that in drafting situation, a cyclist spare about $18 \%$ of oxygen uptake at 32 $\mathrm{km} \cdot \mathrm{h}^{-1}$. The benefit of drafting a single cyclist at 37 and $40 \mathrm{~km} \cdot \mathrm{h}^{-1}$ was greater $(27 \%)$ than at 32 $\mathrm{km} \cdot \mathrm{h}^{-1}$. Drafting one, two, or four cyclists in a line at $40 \mathrm{~km} \cdot \mathrm{h}^{-1}$ resulted in the same reduction in oxygen uptake (27\%). In addition, these authors showed that riding at $40 \mathrm{~km} \cdot \mathrm{h}^{-1}$ at the back of a group of eight cyclists reduced the oxygen uptake by significantly more (39\%) than drafting one, two, or four cyclists in a line.

The emergence of new Olympics sports such open-water swimming (in Beijing 2008) and triathlon (in Sydney 2000) had led sports scientists and national coaches to raise various questions about physiological process regulating these new disciplines. It is obvious that drafting has been studied in each of the three disciplines composing triathlon (Chollet et al. 2000; Kyle, 1979; Pugh, 1971) and has proven far more beneficial to tested subjects compared with swimming and running, as clearly demonstrated by, for instance, the reduction in heart rate when riding in a group (Kyle, 1979). Little is known about drafting in cycling and its influences on the following run during a triathlon. The first interesting finding was given by Hausswirth et al. (1999), indicating that drafting during the bike course of a triathlon (i.e. immediately after the 
swim leg) lowered both energy expenditure, heart rate and pulmonary ventilation values for a drafting distance of 0.2-0.5 m behind a lead cyclist. The authors demonstrated a global reduction oxygen uptake $(-14 \%)$, heart rate $(-7.5 \%)$ and pulmonary ventilation $(-30.8 \%)$ for the drafted bike leg and for an average cycling speed of $39.5 \mathrm{~km} \cdot \mathrm{h}^{-1}$. When we compared these data to the one found by McCole et al. (1990) at a cycling speed of $40 \mathrm{~km} . \mathrm{h}-1$, the reduction in oxygen uptake was about $-26 \%$; the differences in oxygen uptake saved was explained by Hausswirth et al. (1999) with the probably less efficiency at drafting during the initial phase (i.e. first 4-km) of the cycling section of the simulated outdoor triathlon, due to the residual negative effects of the swim stage. The results also showed that running after biking in a drafting situation (for similar bike speeds) significantly improved the running speed compared with that of the no-draft modality (17.8 vs. $17.1 \mathrm{~km} \cdot \mathrm{h}^{-1}$ ). The benefit of drafting in cycling allowed triathletes to push themselves forward in the subsequent run as demonstrated in figure 3. The authors emphasized that drafting during the bike leg of a triathlon creates the conditions for an improved running performance, especially with higher benefits for the strong runners. They concluded that the
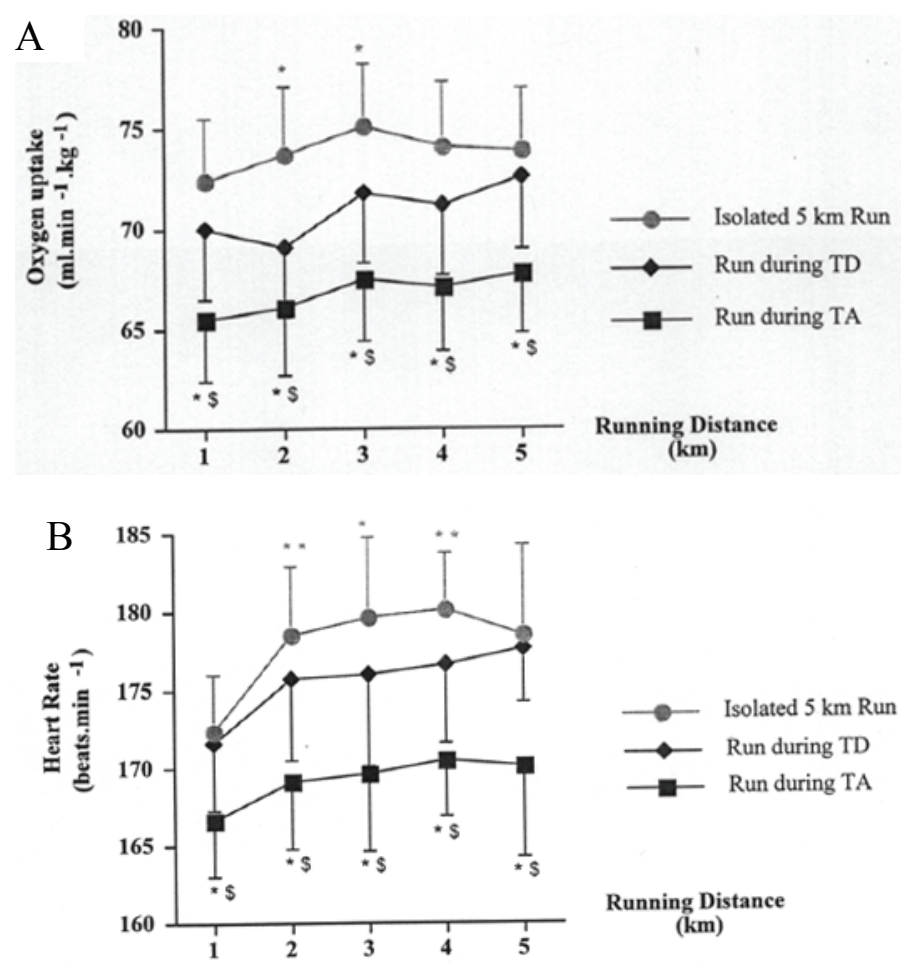

Figure 3 - A, Changes in oxygen uptake values obtained during the run section of drafted (TD) vs. no-drafted (TA) triathlons. B, Changes in heart rate values obtained during the run section of drafted (TD) vs. no-drafted (TA) 
triathlons. ${ }^{*} \mathrm{P}<0.01,{ }^{* *} \mathrm{P}<0.05$, significantly different from Run during TD group, ${ }^{\$} \mathrm{P}<0.01,{ }^{\$} \mathrm{P}<0.05$, significantly different from Run during TA group, (from Hausswirth et al. 1999, reproduced with persmission).

higher expiratory flow observed during the run after drafting in cycling compared with the run after cycling alone may be partly explained the $5.3 \%$ increase in oxygen uptake (Figure 3) stemming from an enhanced oxygen flow to the active muscles. Furthermore, Roberston et al. (1977) suggested that as resistance increases, more energy is needed to generate sufficient tension in the muscles to obtain the pressures required for airflow to occur while some energy is also used to prevent deformation of the chest wall during increase work.Because of the generalization of drafting in cycling during elite triathlon events (i.e. Olympic games) and the various race strategies now induced, it seems important that triathletes know the effects of pacing up with another cyclist in order to save energy for the consecutive run. Within this framework, Hausswirth et al. (2001) investigated more recently the physiological responses of riding alternatively or continuously behind another cyclist during a simulated indoor sprint distance triathlon. The triathlete had to perform two triathlons; one with the alternate drafting process during cycling where the triathlete alternatively rode in front or at the back of another cyclist, rotating every $500-\mathrm{m}$ and keeping the reach speed always constant. In the other modality, each triathlete drafted a professional cyclist whose task was to respect all split times recorded during the alternate bike leg. The authors showed a reduction by $16.5 \%$ in oxygen uptake and $11.4 \%$ in heart rate during the bike leg done continuously compared with the alternate cycling stage. In association, they recorded a better 5-km running performance after the continuous bike leg $(+4.2 \%)$ compared with the run done after the alternate bike leg. The interesting result was the steady-state of the energy cost of running obtained during the runs, due to the increase of running speed after the continuous bike leg in association with a greater oxygen uptake provides by the benefits taken from the previous cycling part. Finally, for elite triathletes who are familiarized with drafting technique in training and world cup triathlon, the run performance depends on the previous cycling event (drafting modalities, pedaling cadence, stochastic power output..) but appealed the same energy cost of running in a sprint distance triathlon.

A form oh human locomotion as yet only poorly studied is speed skating, in which the maximal aerobic speeds are closer to cycling than running $\left(\approx 40 \mathrm{~km} \cdot \mathrm{h}^{-1}\right.$ for the $10,000-\mathrm{m}$ race), 
suggesting that a relevant fraction of the total energy expenditure is spent overcoming air resistance. Di Prampero et al. (1976) quantified the energy spent against wind as being equal for running, skating, and cycling. From this experiment, he concluded that energy spent against forces resulted in the different speeds attained in these exercises for equal power outputs. More recently Rundell (1996a) demonstrated that a short-track race at a speed of $32 \mathrm{~km} . \mathrm{h}^{-1}$ may in fact compromise the benefits of drafting because of internal power losses to overcome high forces required to skate the tight corners. In this light, they indicated most short-track skaters reduced their energy requirements for skating at a constant pace if they drafted another skater. Drafting resulted in a mean of 6 beats. $\min ^{-1}$ reduction in heart rate for the 18 skaters of this study. This difference corresponded to an approximate 5-5.5\% decrease in oxygen uptake (Rundell, 1996b) and is less than expected by the authors, the coefficient of friction for skating is demonstrated to be similar to that of cycling (D Prampero et al. 1976), where drafting at a similar speed resulted in an approximate $31 \%$ reduction in heart rate from leading (Kyle 1979). However, in speedskating the maintenance of an optimal drafting position in corners appears more difficult because synchronous crossing-over of the legs at higher velocities is technically difficult and can lead to slightly higher distance between athletes (Krieg et al. 2006). In addition to speed-skating studies, some investigations have dealt with drafting, although only few investigated cross-country skiing. A study of Bilodeau et al. (1994) clearly indicate that drafting behind another skier could be a major advantage in a race when the situation is encountered. A mean reduction of 9 beats.min-1 was observed when drafting a skier (Figure 4) as compared with leading the same skier, a significant reduction of $5.6 \%$. The estimated energy cost deducted from "Heart rate Oxygen uptake" relationship during the treadmill running test was significantly lower when pacing up with a skier, with a reduction of $13 \%$ when compared in being drafted. Then, the situation where drafting might play a major role advantage in a race are in a pursuit race, during a relay race, and during a mass start race. Moreover, a drafting situation could also happen in an individual start race, when a slower skier is passed, but is capable of pacing up with the slightly faster skier starting the race 30 or $60 \mathrm{~s}$ back. It seems to become a more and more daily practice during training, especially if skiers have to work together by sharing the lead in order to save energy, and thus increase speed.

In running, previous study of Pugh (1970) demonstrated that at a speed of $6 \mathrm{~m} \cdot \mathrm{s}^{-1}, 80 \%$ of the oxygen cost of meeting air resistance was eliminated by running close behind another runner. 
Unless some other adverse effect is present to cancel this advantage, an athlete should be able to exceed the speed corresponding to his maximal oxygen uptake by up to $7.5 \times 0.80=6 \%$, by running behind a pace-maker or a faster competitor. According to the relation of oxygen uptake and speed in track running found by Pugh (1970), the oxygen uptake corresponding to a speed of $6 \mathrm{~m} \cdot \mathrm{s}^{-1}$ is $76 \mathrm{~mL} \cdot \mathrm{min}^{-1} \cdot \mathrm{kg}^{-1}$ and the speed corresponding to a $6 \%$ greater oxygen uptake (i.e. 80.5 $\left.\mathrm{mL} \cdot \mathrm{min}^{-1} \cdot \mathrm{kg}^{-1}\right)$ is $6.4 \mathrm{~m} \cdot \mathrm{s}^{-1}$. This is equivalent of a reduction in time for a 400-m lap from 66.6 to $62.5 \mathrm{sec}$. Track experience, however, suggests that athletes cannot run close enough to gain as much as advantage as this. The reduction in oxygen uptake achieved by running behind another runner at $6 \mathrm{~m} \cdot \mathrm{s}^{-1}$ was $250 \mathrm{~mL} \cdot \mathrm{min}^{-1} \cdot \mathrm{kg}^{-1}$; therefore, by running close behind another runner, oxygen uptake is $6.5 \%$ less than without shielding. Thus $80 \%$ of the energy cost of overcoming air resistance can be abolished by sheltering in running.

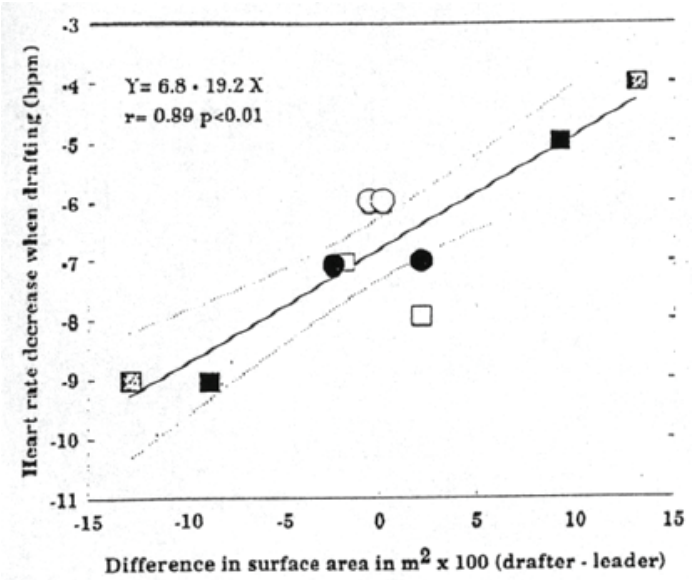

Figure 4 - Relationship between the within-pair difference in average frontal surface area $\left(\mathrm{m}^{2}\right)$ and heart rate decrement while drafting the leading skier. Pairs of similar symbol represent the two skiers of the same pair (regression $\pm 95 \%$ confidence intervals, reproduced with permission from Bilodeau et al. 1995).

\section{- Consequences on biomechanical aspects of performance}

Other previous attempts have been done to explain the determinants of the magnitude of drafting process on focusing on the drafter's skill. Broker et al. (1999) for example showed that the drafting effect, expressed in terms of mass-normalized power output, varied by as much 1.21 W. $\mathrm{kg}^{-1}$ (20.5\% of total power). Previously Kyle (1979) used the four-person team pursuit models 
to find that drafting effect is subject to several skill-related factors - paceline position, interwheels distance between two riders, the drafter's left-right alignment with respect to the leader. Although these researches provide consistent data about drafting effect with respect to its several modalities, most of these informations sets also exhibit extended interindividual variability that can't fully explained skill-related parameters. Very recently, Edwards \& Byrnes (2007) hypothesized that leader drag area is an important determinant of the drafting effect in cycling (see Fig. 5). Therefore they indicated a strong mean effect of leader drag area, whether that effect is expressed in terms of the drag coefficient or power output. In addition, they found that the ratio between drag area of a leader and the drag area of a drafter is strongly correlated with the drafting effect. The pooled correlations indicate that some $61 \%$ of the drafting effect of variance can be accounted for by variation in the leader-drafter drag-area ratio. It seems from these data that the drafter's aerodynamic and anthropometric characteristics have little influence on the measured drafting effects. These results showed a greater mean drafting effects than found by either Kyle et al. (1979, 1988) or broker et al. (1999). Kyle’s 38\% reduction in aerodynamic drag force is approximately 4\% less than the Edwards and Byrnes 's $42.4 \%$ reduction in the drag coefficient. The difference between the power-based of this study estimate of the drafting effect $(33.2 \%)$ and that of Broker et al. (26.9\%) is greater again at 6.3\%. There are several possible explanations for those differences, but the most likely is that the recent study of Edwards and Byrnes (2007) involved a greater mean leader drag than did either of the previous two.

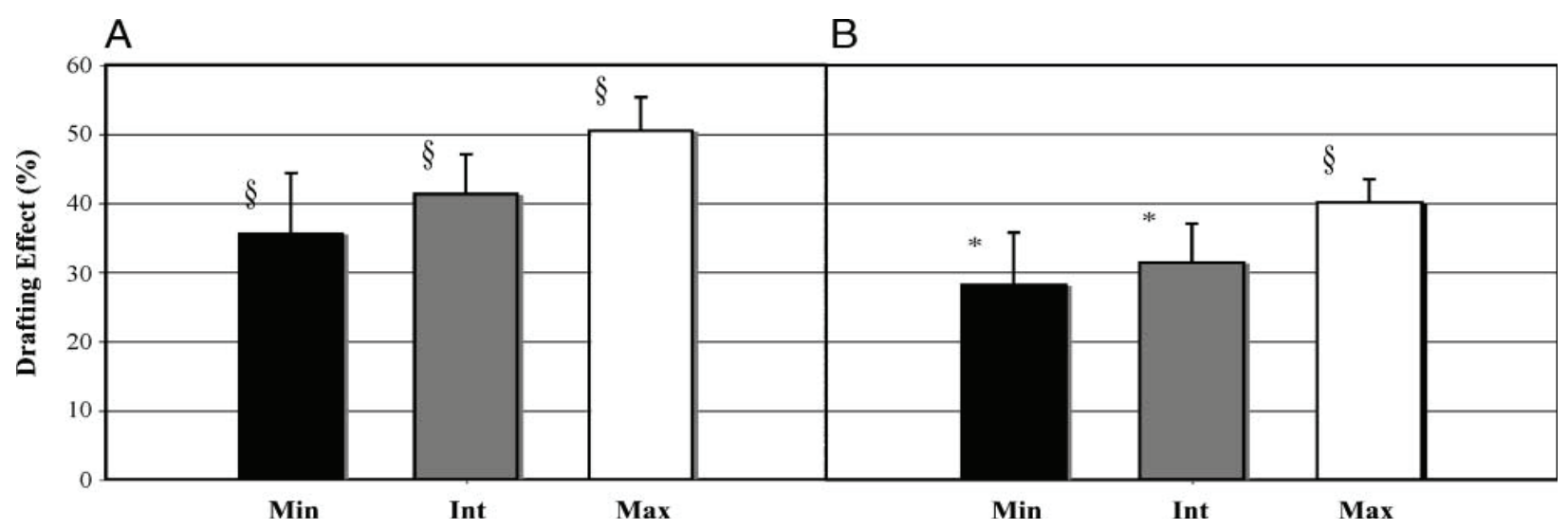

Figure 5 - Depictions of the drafting effect: percent change in drag coefficient (A) or power output (B) from solo condition, observed for the three different leaders (minimum, intermediate, and maximum). ${ }^{*}$ Significantly different from maximum drag-area leader; $\S$ significantly different from both remaining leaders. (from Edwards and Byrnes, 2007). 
As we know, in most endurance sports a large part of the external power delivered by the athlete is used to overcome drag. In level cycling and speed skating the speed is entirely determined by equivalence between external power and frictional losses (di Prampero, 1979). Van Igen Schenau (1982) showed that when a speed-skater is shielded by a subject who is standing in the skating posture 2-m in front of him, the drag is decrease by about 16\%. At 1-m distance this shielding effect cause a decrease in drag of $23 \%$. This effect explains the fast lap times in marathon speed skating in which all competitors skate closely behind each other. In contrast in in-line skating, Millet et al. (2003) demonstrated that the technical difficulties for drafting efficiently, especially while cornering, resulted in a reduced benefit of drafting at high velocity than in other sports. Moreover, the need for the skater to adjust their own cycle frequency to that of the lead skater while drafting "close" would explain partly that there were no significant differences between drafting at about $0.75-\mathrm{m}$ and 1.20-m distances. Moreover, the energy requirement to overcome the high cornering forces could explain why drafting benefits observed were not as great as those observed in cycling. In this context, Rundell (1996) showed for short-track skaters that external power can be estimated from air friction force and ice friction force using the calculated cornering force during short-track and reported values for long-track skating $(\approx 4 \mathrm{~N}$ on the straights and $\approx 6 \mathrm{~N}$ in corners). They found that at $9.2 \mathrm{~m} \cdot \mathrm{s}^{-1}$ the air friction is reduced by $21 \%$ from drafting, allowing a decrease of $13 \%$ in total power.

Skiing constitute another form of locomotion which is well-studied according the process of drafting. For example, Spring et al. (1988) studied the effects of drafting in roller skiing and found that when a skier is in a semisquatting position and pacing up with another skier 2-3 $\mathrm{m}$ ahead, in the same posture, the drag is decreased by about 25\%. Although Street (1990) did not study the effect of drafting, he used Kyle's results (1979) and estimated that skiing at 5.5 m.s ${ }^{-1}$ with no head wind would result approximately a $6 \%$ reduction in total mechanical power. Another of his estimations proposed that skiing with a head wind of $4.5 \mathrm{~m} . \mathrm{s}^{-1}$, the total power output savings for the trailing skier would be about 14\%. Street (1990) also mentioned that the advantages of drafting would occur when flow velocity is large and air drag is the major component of the net resistive force.

Because of the important energy requirement to overcome wind resistance in triathlon especially in cycling and running - the benefits of drafting behind a leader on biomechanical 
changes have been recently investigated. Hausswirth et al. (1999) found the lower energy requirement in the cycling parted of a triathlon where triathlete rode behind a leader compared with alone is linked to higher freely chosen pedaling rate when biking in drafting position (95 rpm) than when biking alone ( $89 \mathrm{rpm})$. The authors suggested that this reduction in applied forces may be explained by a reduction in the activation of the vastus lateralis muscle due to the decrease of wind resistance and connected to a reduction of the energy expenditure. In a more recent study (Hausswirth et al. 20001) simulating an indoor sprint triathlon, the triathletes had to realize two triathlons where during the bike course they 1) alternatively rode in front or at the back of another cyclist (ADT situation), rotating every 500-m and keeping speed always constant, 2) drafted continuously a professional road cyclist (CDT situation) who task was to respect all split times recorded during ADT situation by the triathlete they were sheltering. The results showed that triathletes adopted higher freely chosen cadences during ADT (102 rpm) compared with CDT situation ( $85 \mathrm{rpm}$ ). The authors suggested in the context of triathlon races

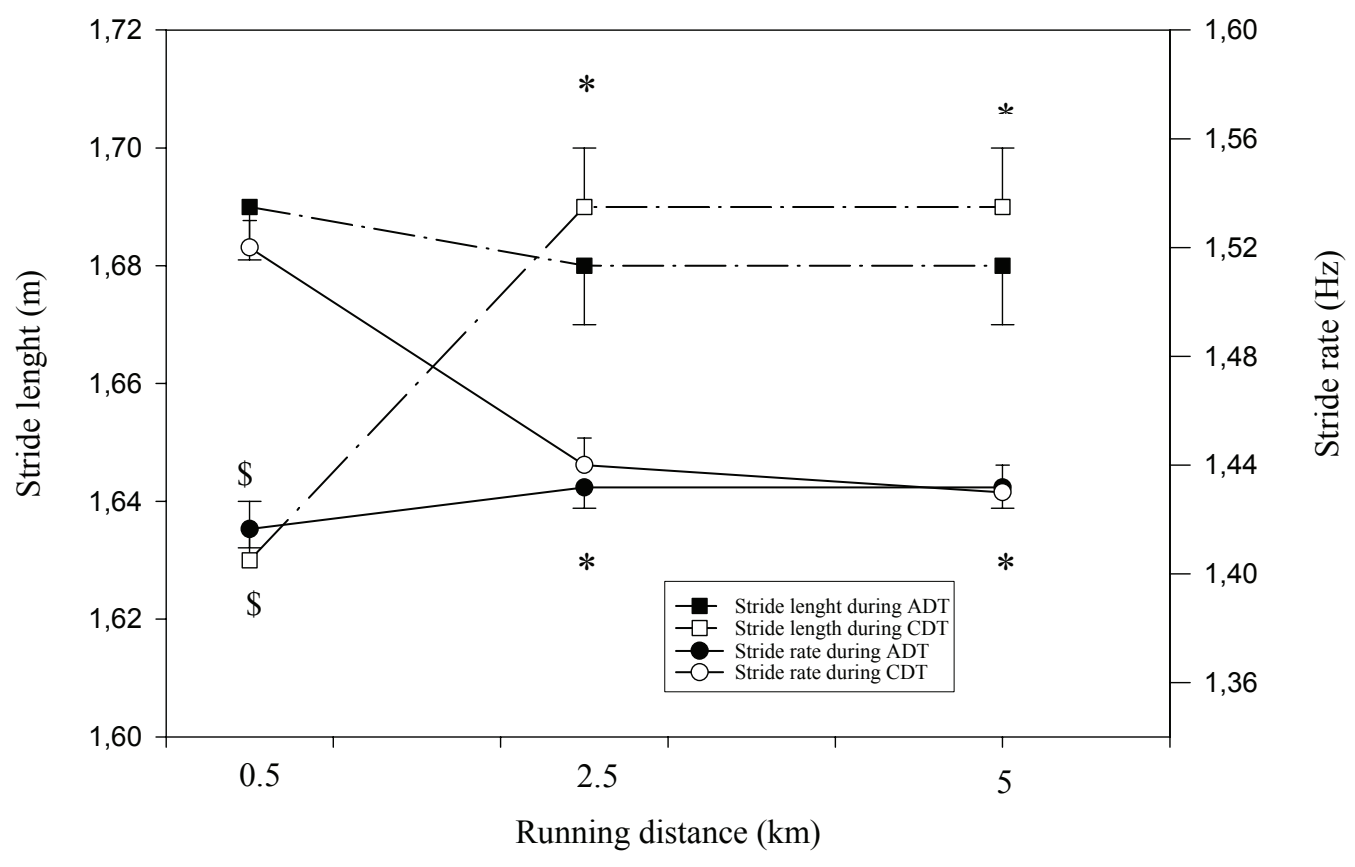

Figure 6 - Changes in stride length and stride rate values obtained during the run section of the alternate draft triathlon (ADT) vs. the continuous draft triathlon (CDT). Significantly different from the initial value, ${ }^{*} \mathrm{P}<0.05$. 
Significantly different from the corresponding ADT value, ${ }^{\$} \mathrm{P}<0.05$, (from Hausswirth et al. 2001, reproduced with permission).

the necessity to implement drafting techniques in training to save energy for the final run associated with the stride length triathletes adopted immediately after the bike leg (see Figure 6). At the beginning of the run done after biking in ADT situation, the stride was shorter compared with the CDT run value ( $1.63 \mathrm{~m}$ vs. $1.68 \mathrm{~m}$, respectively). The authors showed that the pedaling cadence in cycling influence the stride rate in running only during the part of the run, as evidenced by the lack of change in stride length and stride rate recorded from the $2^{\text {nd }}$ to the $5^{\text {th }}$ $\mathrm{km}$ run (figure 6). Tehn, similar stride length values were obtained from the middle to the end of the final run section of both triathlons (CDT and ADT), suggesting with exercise duration the triathlete adopted spontaneously the same pattern of locomotion, i.e. the one eliciting the lowest energy cost of running.

To conclude, during multiple endurance sports events athletes have the opportunity to draft one another. Drafting is well-known to limit the aerodynamic resistive force they experience, and affords less physiologically capable individuals the ability to maintain the pace of their more capable counterparts and, in doing so, adds complexity to the prediction of racing performance. Indeed, the respite offered by drafting is the single factor that predisposes mass-start races (i.e. triathlon, road cycling, skiing, short-track skating, kayaking...) to a degree of tactical complexity not apparent in individual time-trial competitions. Finally, the practice of drafting would be incorporated in training programs in order to optimize all physiological and biomechanical adaptations occurring when athletes trained. 


\section{REFERENCES}

1. Bassett DR, Flohr J, Duey WJ, Howley ET, \& Pein RL (1991). Metabolic responses to drafting during front crawl swimming. Med Science Sports Exer 23: 744-747.

2. Bentley DJ, Libicz S, Jougla A, Coste O, Manetta J, Chamari K, \& Millet GP (2007). The effects of exercise intensity or drafting during swimming on subsequent cycling performance in triathletes. $J$ Sci Med Sport 10:234-243.

3. Bentley DJ, Millet GP, Vleck VE, \& MacNaughton LR (2002). Specific aspects of contempory triathlon. Sports Med. 32:1-15.

4. Broker JP, Kyle CR, \& Burke ER (1999). Racing cyclist power requirements in the 4000$\mathrm{m}$ individual and team pursuits. Med Sci Sports Exerc 31:1677-1685.

5. Chatard JC \& Millet GP (1996). Effects of wetsuit use in swimming events. Sports Med 22 : 70-75.

6. Chatard JC, \& Wilson B (2003). Drafting distance in swimming. Med Science Sports Exer 35: 1176-1181.

7. Chatard, JC, Chollet D, \& Millet GP (1998). Performance and drag during drafting swimming in highly trained triathletes. Med Science Sports Exer 30: 1276-1280.

8. Chollet D, Hue O, Auclair F, Millet GP, \& Chatard JC. (2000). The effects of drafting on stroking variations during swimming in elite male triathletes. Eur J Appl Physiol 82:413417.

9. Delextrat A, Tricot V, Bernard T, Vercruyssen F, Hausswirth C; \& Brisswalter J (2003). Drafting during swimming improves efficiency during subsequent cycling. Med Sci Sports Exer 35, 1612-1619

10. Di Prampero PE (2000). Cycling on earth, in space, on the moon. Eur J Appl Physiol 82, 345-360.

11. Edwards AG, \& Byrnes WC (2007). Aerodynamics characteristics as determinants of the drafting effect in cycling. Med Sci Sports Exerc 39:170-176.

12. Hausswirth C, Lehénaff D, Dreano P, \& Savonen K (1999). Effects of cycling alone or in a sheltered position on subsequent running performance during a triathlon. Med. Sci. Sports Exerc. 31:599-604. 
13. Hausswirth C, Vallier JM, Lehénaff D, Brisswalter J, Smith D, Millet G, \& Dreano P (2001). Effect of two drafting modalities in cycling on running performance. Med Sci Sports Exerc 33:385-390.

14. Jeukendrup AE, Craig NP, \& Hawley JA (2000). The bioenergetics of world class cycling. J Sci Med Sport 3:414-433.

15. Kreider RB, Cundiff DJ, Hammet JB, Cortes CW, \& Williams KW (1988). Effects of cycling on running performance in triathletes. Annals of Sports Med 3, 220-225.

16. Kyle, CR (1979). Reduction of wind resistance and power output of racing cyclists and runners travelling in groups. Ergonomics 22: 387-397.

17. Laursen PB, Rhodes EC, \& Langill RH (2000). The effects of 3000-m swimming on subsequent 3-h cycling performance: implications for ultraendurance triathletes. Eur $J$ Appl Physiol 83: 28-33.

18. McCole SD, Claney K, Conte JC, Anderson R, \& Hagberg JM (1990). Energy expenditure during bicycling. J Appl Physiol 68:748-753.

19. Millet GP, Chollet D, \& Chatard JC (2000). Effects of drafting behind a two- or a sixbeat kick swimmer in elite female triathletes. Eur J Appl Physiol 82: 465-471.

20. Olds T (2001). Modelling human locomotion: applications to cycling. Sports Med 31:497-509.

21. Pugh LG (1970). Oxygen intake in track and treadmill running with observations on the effect of air resistance. J Physiol 207, 823-835.

22. Pugh LG (1971). The influence of wind resistance in running and walking and the mechanical efficiency of work against horizontal or vertical forces. J Physiol 213, 255276.

23. Sargent RM (1926). The relation between oxygen requirement and speed in running. Proc R Soc 100:10-22.

24. Vleck V, Bürgi A, \& Bentley DJ (2006). The consequences of swim, cycle, and run performance on overall result in elite Olympic distance triathlon. Int J Sports Med 27:4348. 